\title{
COSMIC RECYCLING OF MILLISECOND PULSARS
}

\author{
Wynn C. G. Ho ${ }^{1}$, Thomas J. Maccarone ${ }^{2}$, and Nils Andersson ${ }^{1}$ \\ ${ }^{1}$ School of Mathematics, University of Southampton, Southampton, SO17 1BJ, UK; wynnho@slac.stanford.edu, na@maths.soton.ac.uk \\ ${ }^{2}$ School of Physics \& Astronomy, University of Southampton, Southampton, SO17 1BJ, UK; tjm@phys.soton.ac.uk \\ Received 2010 December 8; accepted 2011 February 18; published 2011 March 14
}

\begin{abstract}
We compare the rotation rate of neutron stars in low-mass X-ray binaries (LMXBs) with the orbital period of the binaries. We find that, while short orbital period LMXBs span a range of neutron star rotation rates, all the long-period LMXBs have fast rotators. We also find that the rotation rates are highest for the systems with the highest mean mass accretion rates, as can be expected if the accretion rate correlates with the orbital period. We show that these properties can be understood by a balance between spin-up due to accretion and spin-down due to gravitational radiation. Our scenario indicates that the gravitational radiation emitted by these systems may be detectable by future ground-based gravitational wave detectors.
\end{abstract}

Key words: accretion, accretion disks - gravitational waves - pulsars: general - stars: evolution - stars: neutron $\mathrm{X}$-rays: binaries

Online-only material: color figures

\section{INTRODUCTION}

Accreting millisecond X-ray pulsars are the evolutionary link between the (million-year old) classical radio pulsars and the (billion-year old) millisecond pulsars (Smarr \& Blandford 1976; Alpar et al. 1982; Radhakrishnan \& Srinivasan 1982). The accreting millisecond pulsars (AMSPs) are found in lowmass X-ray binaries (LMXBs; Chakrabarty \& Morgan 1998; Wijnands \& van der Klis 1998), systems in which the pulsar is accreting from a low-mass stellar companion. The details of how the transition from a rotating neutron star in an LMXB to a millisecond radio pulsar takes place are unknown (Bhattacharya 1995), although a system has been found recently which shows evidence for having made this transition (Archibald et al. 2009).

We show here that a significant clue regarding the transition may be contained in the relation between the rotation rate and orbital period for the known AMSPs. This trend suggests that a common physical mechanism(s) may be responsible. We outline a simple scenario that can explain the evolution of the rotation rate and orbital period for AMSPs and show how this naturally leads to the observed population. The proposed evolutionary scenario predicts gravitational wave emission at a level that may be detected by next-generation ground-based observatories.

\section{DATA}

We use the tabulated values for the most rapidly rotating $\left(v_{\mathrm{s}} \geqslant 100 \mathrm{~Hz}\right)$ AMSP spin frequency $v_{\mathrm{s}}$, orbital period $P_{\text {orb }}$, and mass accretion rate $\dot{M}$ from Galloway (2008) and Watts et al. (2008). ${ }^{3}$ The spin frequencies of some AMSPs are derived from brightness oscillations seen during X-ray bursts (Strohmayer et al. 1996; Chakrabarty et al. 2003), which are due to thermonuclear burning of accreted material on the surface of the neutron star (Strohmayer \& Bildsten 2006). We do not consider spin frequencies derived from $\mathrm{kHz}$ quasi-periodic oscillations since the correlation between the spin and quasiperiodic oscillation frequencies is uncertain (Wijnands et al. 2003).

\footnotetext{
3 Note that Galloway et al. (2008) and Watts et al. (2008) state that the orbital period of $4 \mathrm{U} 1702-429$ is unknown.
}

The trend in the measured values of the spin frequency and orbital period for the AMSPs is shown in Figure 1. Also shown for comparison are values for the known (rotation-powered) millisecond radio pulsars from the ATNF Pulsar Catalogue ${ }^{4}$ (Manchester et al. 2005); these rotation-powered pulsars are older and thought to be the descendants of the AMSPs. While the rotation-powered pulsars are distributed fairly uniformly in the diagram of $v_{\mathrm{s}}$ versus $P_{\text {orb }}$, the AMSPs are not. In particular, at the highest rotation rates, the orbital periods of the AMSPs span the range from about $0.5 \mathrm{hr}$ to $20 \mathrm{hr}$, but there are no AMSPs with $100 \mathrm{~Hz} \lesssim v_{\mathrm{s}}<440 \mathrm{~Hz}$ and $P_{\text {orb }}>4.3 \mathrm{hr}$. A similar plot to Figure 1 was presented by Kaaret et al. (2006), who remarked upon a possible absence of slowly-rotating, long orbital period systems; they suggested that this may be due to an accretion rate that varies with orbital period but made no further discussion. A full population synthesis of neutron star spin and orbital periods is beyond the scope of this work. Nevertheless, a qualitative explanation of the $v_{\mathrm{s}}-P_{\text {orb }}$ distribution of AMSPs can be obtained by invoking simple physical processes that occur in their evolution.

\section{AMSP EVOLUTION}

In close binary systems such as the LMXBs, matter near the surface of the companion star is transferred to the primary star (the latter is a neutron star in the case of AMSPs) when the companion radius $R_{\mathrm{c}}$ is equal to the Roche lobe size $R_{\mathrm{L}}$. This size is the distance from the center of the donor star to the inner Lagrangian point, where the gravitational forces from the companion and primary are equal and opposite, and is given by (Paczyński 1971)

$$
R_{\mathrm{L}} \approx 0.46 a\left[m_{\mathrm{c}} /\left(m_{\mathrm{x}}+m_{\mathrm{c}}\right)\right]^{1 / 3},
$$

where $a$ is the orbital separation between the neutron star of mass $m_{\mathrm{x}}$ and the companion star of mass $m_{\mathrm{c}}\left(m_{\mathrm{x}}\right.$ and $m_{\mathrm{c}}$ are in units of solar mass $M_{\odot}$ ); hereafter, we assume $m_{\mathrm{x}}=1.4$. When the companion is a main-sequence star, $m_{\mathrm{c}} \approx R_{\mathrm{c}} / R_{\odot}$, where

\footnotetext{
4 http://www.atnf.csiro.au/research/pulsar/psrcat
} 


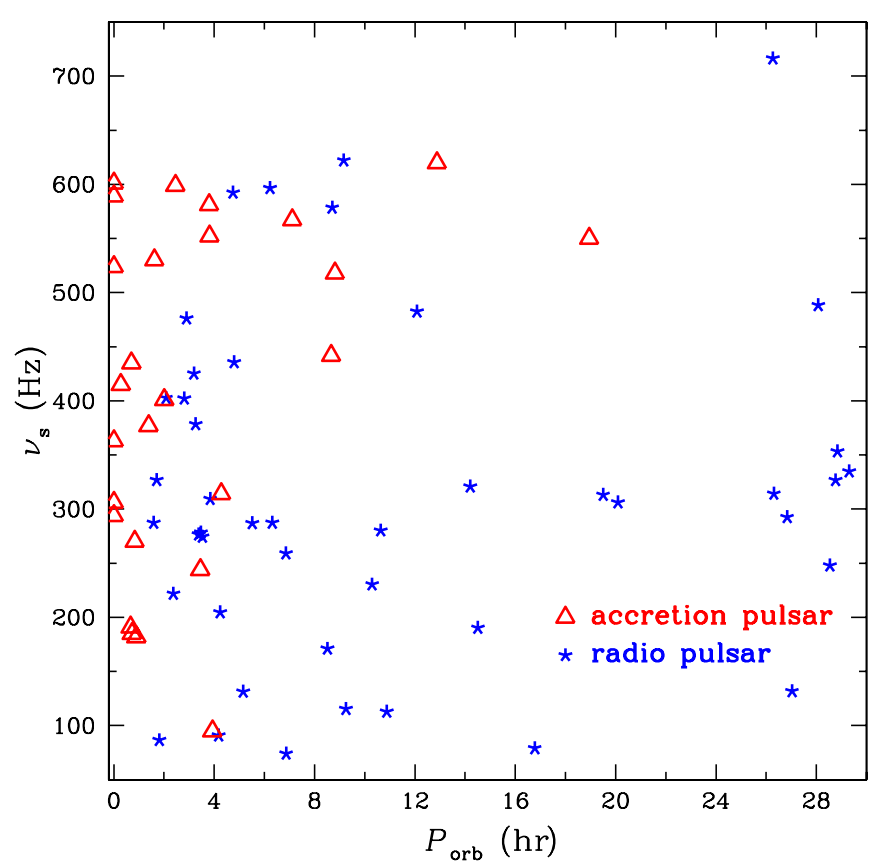

Figure 1. Observed spin frequency as a function of orbital period for the accreting millisecond pulsars (triangles) and rotation-powered radio pulsars (stars). The spins and orbits for the accreting millisecond pulsars are from Galloway (2008) and Watts et al. (2008), while those for the radio pulsars are from the ATNF Pulsar Catalogue (Manchester et al. 2005).

(A color version of this figure is available in the online journal.)

$R_{\odot}$ is the solar radius (Verbunt \& van den Heuvel 1995). Using Kepler's third law,

$$
a=0.8 R_{\odot}\left(m_{\mathrm{x}}+m_{\mathrm{c}}\right)^{1 / 3}\left(P_{\mathrm{orb}} / 2 \mathrm{hr}\right)^{2 / 3},
$$

we obtain a relationship between the mass of the companion and the orbital period (King et al. 1996),

$$
m_{\mathrm{c}}=0.23 \hat{m}_{\mathrm{c}}\left(P_{\mathrm{orb}} / 2 \mathrm{hr}\right),
$$

where $\hat{m}_{\mathrm{c}} \equiv m_{\mathrm{c}} / m_{\mathrm{c}}^{\mathrm{MS}}$ and $m_{\mathrm{c}}^{\mathrm{MS}}$ is the mass of a main-sequence star that just fills its Roche lobe at $P_{\text {orb }}$; the factor of $\hat{m}_{\mathrm{c}}(<1)$ is due to the fact that the companion star in a binary has a larger radius for its mass than an isolated star (see, e.g., Sills et al. 2000; Andronov et al. 2003) and encapsulates our uncertainty in the (less evolved, since we only consider $m_{\mathrm{c}}<m_{\mathrm{x}}$ ) evolutionary state of the companion.

We now examine the various physical processes that would lead to a change in the angular momentum of the orbit or neutron star spin and thus cause a source to move within the $v_{\mathrm{s}}-P_{\text {orb }}$ plane of Figure 1. Mass transfer from the companion to the neutron star can cause an increase in the size of the orbit, while orbital angular momentum loss due to magnetic braking or gravitational wave emission causes the orbit to decrease. The orbital period which separates expansion and decay is estimated to be $>0.5$ days (see, e.g., Podsiadlowski et al. 2002; Ma \& Li 2009). For orbital periods longer than $1 \mathrm{hr}$, magnetic braking is dominant. We consider two prescriptions for the magnetic braking torque. The first one, from Verbunt \& Zwaan (1981), results in a timescale for decay

$$
\tau_{\text {decay }}^{\mathrm{VZ}} \approx\left(2.1 \times 10^{8} \mathrm{yr}\right) m_{\mathrm{x}}^{2 / 3} \hat{m}_{\mathrm{c}}^{-4 / 3}\left(P_{\text {orb }} / 2 \mathrm{hr}\right)^{-2 / 3}
$$

and a mass transfer rate $\dot{M}_{-11}$ (in units of $10^{-11} M_{\odot} \mathrm{yr}^{-1}$ ) (see also King et al. 1996)

$$
\dot{M}_{-11}^{\mathrm{VZ}}=80 m_{\mathrm{x}}^{-2 / 3} \hat{m}_{\mathrm{c}}^{7 / 3}\left(P_{\mathrm{orb}} / 2 \mathrm{hr}\right)^{5 / 3} .
$$

However, observations of rapidly rotating low-mass stars in open clusters suggest that the rate of angular momentum loss described in Verbunt \& Zwaan (1981) is too high (Sills et al. 2000; Andronov et al. 2003; see also Yungelson \& Lasota 2008, for more discussion). Therefore, we also use the magnetic braking torque from Chaboyer et al. (1995) and Sills et al. (2000) (see also Andronov et al. 2003; Ma \& Li 2009), which results in

$$
\begin{gathered}
\tau_{\text {decay }}^{\mathrm{CS}} \approx\left(2.0 \times 10^{8} \mathrm{yr}\right) m_{\mathrm{x}}^{2 / 3} \hat{m}_{\mathrm{c}}\left(P_{\text {orb }} / 2 \mathrm{hr}\right)^{7 / 3} \\
\dot{M}_{-11}^{\mathrm{CS}}=87 m_{\mathrm{x}}^{-2 / 3}\left(P_{\mathrm{orb}} / 2 \mathrm{hr}\right)^{-4 / 3} .
\end{gathered}
$$

Note that the mass accretion rate given by Equation (5) increases with orbital period, whereas that given by Equation (7) decreases. We also note that, at very short orbital periods $P_{\text {orb }} \lesssim$ $4 \mathrm{hr}$, gravitational radiation becomes important and some AMSP companions are degenerate stars. These systems may then be evolving to longer orbital periods, but they will evolve slowly because the mass transfer rate and angular momentum loss rate are low. For example, SAX J1808.4-3658 has a degenerate companion and increasing orbital period with timescale $P_{\text {orb }} / \dot{P}_{\text {orb }} \approx 6 \times 10^{7}$ yr (Podsiadlowski et al. 2002; Di Salvo et al. 2008; Hartman et al. 2008, 2009).

Accretion of angular momentum-carrying matter from the companion can spin up the neutron star. The timescale for spinup is $\tau_{\text {su }} \approx\left(1.5 \times 10^{9} \mathrm{yr}\right) m_{\mathrm{x}}^{-3 / 7} B_{8}^{-2 / 7} \dot{M}_{-11}^{-6 / 7}\left(v_{\mathrm{s}} / 100 \mathrm{~Hz}\right)$, where $B_{8}$ is the neutron star magnetic field (in units of $10^{8} \mathrm{G}$ ). Using the mass accretion rate from Equations (5) and (7), we obtain

$$
\begin{aligned}
\tau_{\mathrm{su}}^{\mathrm{VZ}} & =\left(3.4 \times 10^{7} \mathrm{yr}\right) m_{\mathrm{x}}^{1 / 7} B_{8}^{-2 / 7} \hat{m}_{\mathrm{c}}^{-2}\left(P_{\mathrm{orb}} / 2 \mathrm{hr}\right)^{-10 / 7}\left(v_{\mathrm{s}} / 100 \mathrm{~Hz}\right) \\
\tau_{\mathrm{su}}^{\mathrm{CS}} & =\left(3.2 \times 10^{7} \mathrm{yr}\right) m_{\mathrm{x}}^{1 / 7} B_{8}^{-2 / 7}\left(P_{\mathrm{orb}} / 2 \mathrm{hr}\right)^{8 / 7}\left(v_{\mathrm{s}} / 100 \mathrm{~Hz}\right) .
\end{aligned}
$$

Spin-down of the neutron star by gravitational quadrupole radiation occurs on a timescale

$$
\tau_{\mathrm{sd}} \approx\left(2.9 \times 10^{10} \mathrm{yr}\right) m_{\mathrm{x}}^{-2} \varepsilon_{-8}^{-2}\left(v_{\mathrm{s}} / 100 \mathrm{~Hz}\right)^{-4},
$$

where $\varepsilon=10^{-8} \varepsilon_{-8}$ is the quadrupole ellipticity.

We ignore spin-down by electromagnetic dipole radiation, which only becomes dominant at $B_{8} \gtrsim 10 \varepsilon_{-8}\left(v_{\mathrm{s}} / 100 \mathrm{~Hz}\right)$. It is instructive to estimate the magnetic field that would be required for the AMSPs to be in magnetic spin equilibrium. Magnetic spin equilibrium occurs when the rotation period of the neutron star is equal to the Keplerian period of the inner accretion disk at the magnetosphere boundary (Davidson \& Ostriker 1973); this leads to an equilibrium spin period

$$
\nu_{\mathrm{s}}^{\mathrm{eq}}=(270 \mathrm{~Hz}) m_{\mathrm{x}}^{5 / 7} B_{8}^{-6 / 7} \dot{M}_{-11}^{3 / 7}
$$

or magnetic field

$$
B^{\mathrm{eq}}=\left(3.2 \times 10^{8} \mathrm{G}\right) m_{\mathrm{x}}^{5 / 6} \dot{M}_{-11}^{1 / 2}\left(v_{\mathrm{s}} / 100 \mathrm{~Hz}\right)^{-7 / 6} .
$$

Neutron stars with $v_{\mathrm{s}}>\nu_{\mathrm{s}}^{\mathrm{eq}}\left(\right.$ or $B>B^{\mathrm{eq}}$ ) can act as mass propellers, as their rapid rotation creates a centrifugal barrier to accretion, while stars with $v_{\mathrm{s}}<v_{\mathrm{s}}^{\mathrm{eq}}$ can accrete mass, gain angular momentum, and be spun-up.

We consider whether the observed properties of neutron stars are consistent with the idea that they are in magnetic spin equilbrium. First, AMSPs are likely to have $B<B^{\text {eq }}$ (Hartman et al. 2009, 2011; Papitto et al. 2010; Patruno 2010). Using the maximum accretion rate observed during outbursts (Galloway 2008), the high implied $B^{\text {eq }}$ (see Figure 2) could channel the 


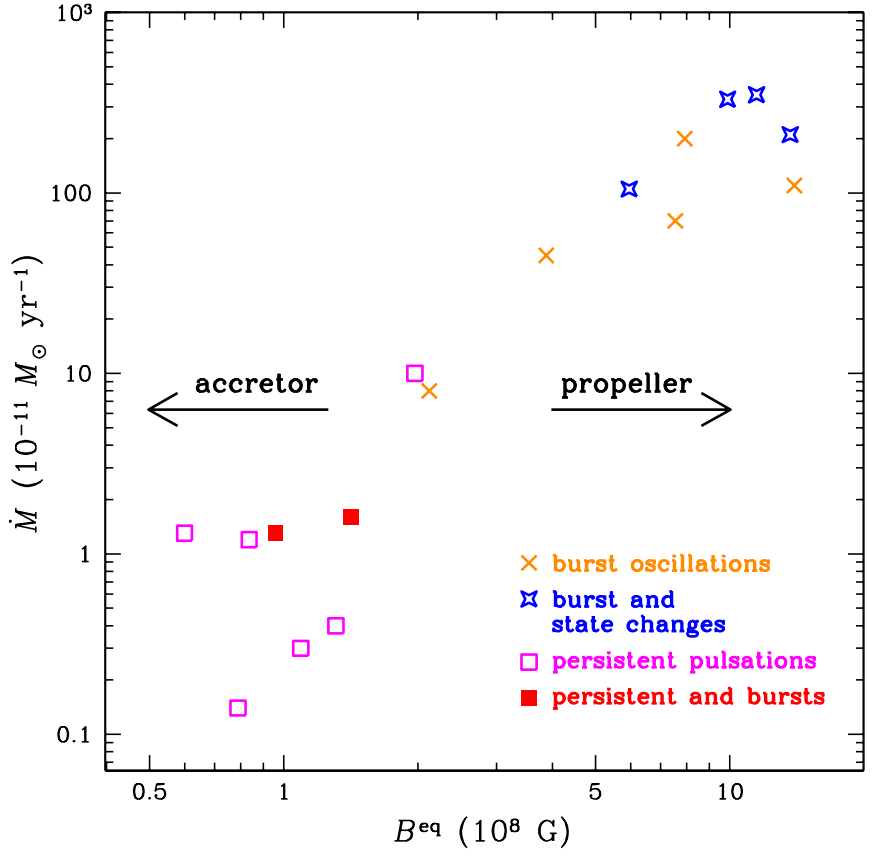

Figure 2. Magnetic field of accreting millisecond pulsars obtained from assuming the neutron star rotates at spin equilibrium (see the text) for the observed mass accretion rate. For a given $\dot{M}$, neutron stars with $B>B^{\text {eq }}$ are mass propellers, while stars with $B<B^{\text {eq }}$ are accretors.

(A color version of this figure is available in the online journal.)

accretion flow and produce persistent coherent pulsations that are not detected; the absence of persistent pulsations in the high accretion rate systems, where the spin is generally measured from burst oscillations, could be the result of magnetic screening by the accreted material (Cumming et al. 2001). In addition, some systems do not show changes to a propeller state at the accretion rates estimated from spin equilibrium (Barret \& Olive 2002; Maccarone \& Coppi 2003; Gladstone et al. 2007).

The evolution of $v_{\mathrm{s}}$ and $P_{\mathrm{orb}}$ is primarily determined by the process with the shortest timescale. A comparison of the timescales is shown in Figures 3 and 4. Over the ranges displayed, there are three regions/regimes: spin-up from the accretion torque (see Equation (8)), spin-down from gravitational quadrupole radiation (see Equation (9)), and orbit decay from magnetic braking (see Equation (4) or (6)). An AMSP moves to higher $v_{\mathrm{s}}$ (spin-up regime) if the pulsar rotation rate is below both $v_{1}$ (from $\tau_{\text {su }}<\tau_{\text {sd }}$ ) and $v_{3}$ (from $\tau_{\text {su }}<\tau_{\text {decay }}$ ), where $v_{1}$ is given by

$$
\begin{aligned}
& v_{1}^{\mathrm{VZ}}=(330 \mathrm{~Hz}) \varepsilon_{-8}^{-2 / 5} B_{8}^{2 / 35} \hat{m}_{\mathrm{c}}^{2 / 5}\left(P_{\mathrm{orb}} / 2 \mathrm{hr}\right)^{2 / 7} \\
& v_{1}^{\mathrm{CS}}=(340 \mathrm{~Hz}) \varepsilon_{-8}^{-2 / 5} B_{8}^{2 / 35}\left(P_{\mathrm{orb}} / 2 \mathrm{hr}\right)^{-8 / 35}
\end{aligned}
$$

and $\nu_{3}$ is given by

$$
\begin{aligned}
& v_{3}^{\mathrm{VZ}}=(750 \mathrm{~Hz}) B_{8}^{2 / 7} \hat{m}_{\mathrm{c}}^{2 / 3}\left(P_{\text {orb }} / 2 \mathrm{hr}\right)^{16 / 21} \\
& v_{3}^{\mathrm{CS}}=(740 \mathrm{~Hz}) B_{8}^{2 / 7} \hat{m}_{\mathrm{c}}\left(P_{\text {orb }} / 2 \mathrm{hr}\right)^{25 / 21} .
\end{aligned}
$$

If the rotation rate is above $\nu_{1}$ and $\nu_{2}$ (from $\tau_{\text {sd }}<\tau_{\text {decay }}$ ), where $v_{2}$ is given by

$$
\begin{aligned}
& v_{2}^{\mathrm{VZ}}=(270 \mathrm{~Hz}) \varepsilon_{-8}^{-1 / 2} \hat{m}_{\mathrm{c}}^{1 / 3}\left(P_{\mathrm{orb}} / 2 \mathrm{hr}\right)^{1 / 6} \\
& v_{2}^{\mathrm{CS}}=(280 \mathrm{~Hz}) \varepsilon_{-8}^{-1 / 2} \hat{m}_{\mathrm{c}}^{-1 / 4}\left(P_{\mathrm{orb}} / 2 \mathrm{hr}\right)^{-7 / 12},
\end{aligned}
$$

then the AMSP moves to lower $v_{\mathrm{s}}$ (spin-down regime). Finally, a decrease in orbital period (orbit decay regime) occurs when the

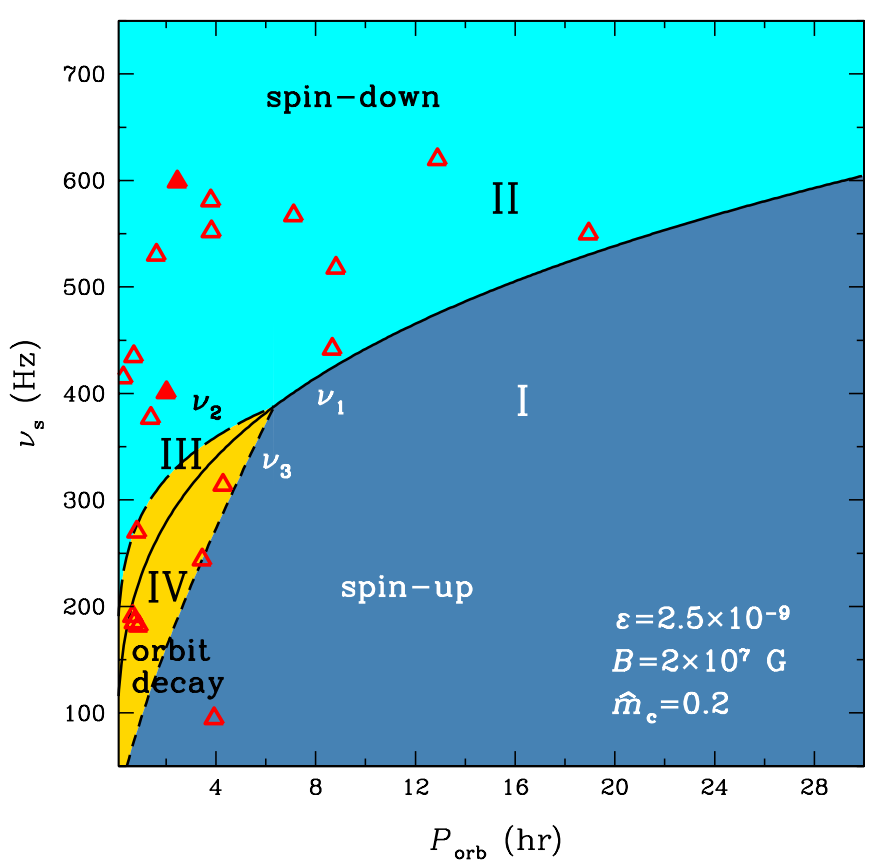

Figure 3. Evolution regimes of accreting millisecond pulsars: AMSPs in the spin-up/spin-down regime (I/II) move to higher/lower spin frequencies, as well as to shorter orbital periods but on a longer timescale; AMSPs in the orbit decay regime (III/IV) move to shorter orbital periods, as well as to lower/higher spin frequencies but on a longer timescale. The lines (labeled $v_{1}^{\mathrm{VZ}}, v_{2}^{\mathrm{VZ}}, v_{3}^{\mathrm{VZ}}$ ) separating the regimes are given by Equations (12)-(14). The open triangles denote AMSPs, while the solid triangles are the two AMSPs, SAX J1808.4-3658 at $\left(P_{\text {orb }}, v_{\mathrm{s}}\right)=(2 \mathrm{hr}, 401 \mathrm{~Hz})$ and IGR J00291+5934 at $(2.5 \mathrm{hr}, 599 \mathrm{~Hz})$, which show short-term spin-up and long-term spin-down, respectively (see the text).

(A color version of this figure is available in the online journal.)

rotation rate is below $v_{2}$ and above $v_{3}$. We note that deviations from low values of $\hat{m}_{\mathrm{c}}$ (up to $\sim 1$, e.g., due to degenerate companions; King et al. 1996) have the strongest effect on $v_{3}$, which do not change our results qualitatively.

Though dependent on the various parameters, the absolute timescale of the dominant process in each regime bears out the observed population, with a slight preference for a $\tau_{\mathrm{su}}$ that decreases with $P_{\text {orb }}$, such as $\tau_{\text {su }}^{\mathrm{VZ}}$ (which is due to a magnetic braking torque that scales with $P_{\text {orb }}^{\gamma}$, where $\gamma>1 / 3$ ). In the spin-up region (long $P_{\text {orb }}$, low $v_{\mathrm{s}}$ ),

$$
\begin{aligned}
\tau_{\mathrm{su}}^{\mathrm{VZ}} & \sim\left(7 \times 10^{6} \mathrm{yr}\right) \hat{m}_{\mathrm{c}}^{-2}\left(P_{\mathrm{orb}} / 10 \mathrm{hr}\right)^{-10 / 7}\left(v_{\mathrm{s}} / 200 \mathrm{~Hz}\right) \\
\tau_{\mathrm{su}}^{\mathrm{CS}} & \sim\left(4 \times 10^{8} \mathrm{yr}\right)\left(P_{\mathrm{orb}} / 10 \mathrm{hr}\right)^{8 / 7}\left(v_{\mathrm{s}} / 200 \mathrm{~Hz}\right) .
\end{aligned}
$$

In the spin-down region (high $v_{\mathrm{s}}$ ), evolution is independent of orbital period and occurs on a timescale

$$
\tau_{\mathrm{sd}} \sim\left(2 \times 10^{9} \mathrm{yr}\right)\left(\varepsilon / 10^{-9}\right)^{-2}\left(\nu_{\mathrm{s}} / 500 \mathrm{~Hz}\right)^{-4} .
$$

In the orbit decay region (short $P_{\text {orb }}$ ), evolution is independent of spin frequency and occurs on a timescale

$$
\begin{aligned}
& \tau_{\text {decay }}^{\mathrm{VZ}} \sim\left(2 \times 10^{8} \mathrm{yr}\right) \hat{m}_{\mathrm{c}}^{-4 / 3}\left(P_{\text {orb }} / 3 \mathrm{hr}\right)^{-2 / 3} \\
& \tau_{\text {decay }}^{\mathrm{CS}} \sim\left(6 \times 10^{8} \mathrm{yr}\right) \hat{m}_{\mathrm{c}}\left(P_{\text {orb }} / 3 \mathrm{hr}\right)^{7 / 3} .
\end{aligned}
$$

The two AMSPs, SAX J1808.4-3658 (Hartman et al. 2009) and IGR J00291 + 5934 (Falanga et al. 2005; Papitto et al. 2010; Patruno 2010; Hartman et al. 2011), show an overall spin-down with timescales $v_{\mathrm{s}} /\left|\dot{v}_{\mathrm{s}}\right| \approx 2 \times 10^{10} \mathrm{yr}$ and $5 \times 10^{9} \mathrm{yr}$, respectively (interrupted by short spin-ups during outbursts with timescales $10^{8} \mathrm{yr}$ and $2 \times 10^{7} \mathrm{yr}$, respectively). 


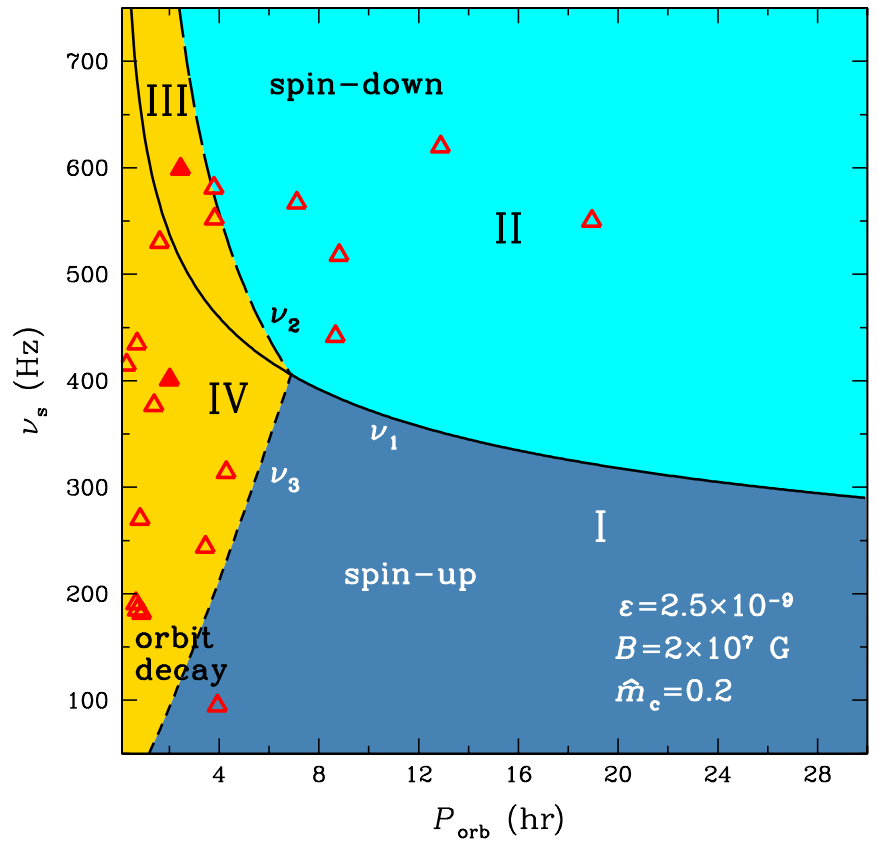

Figure 4. Evolution regimes of accreting millisecond pulsars: AMSPs in the spin-up/spin-down regime (I/II) move to higher/lower spin frequencies, as well as to shorter orbital periods but on a longer timescale; AMSPs in the orbit decay regime (III/IV) move to shorter orbital periods, as well as to lower/higher spin frequencies but on a longer timescale. The lines (labeled $v_{1}^{\mathrm{CS}}, v_{2}^{\mathrm{CS}}, v_{3}^{\mathrm{CS}}$ ) separating the regimes are given by Equations (12)-(14). The open triangles denote AMSPs, while the solid triangles are the two AMSPs, SAX J1808.4 3658 at $\left(P_{\mathrm{orb}}, v_{\mathrm{s}}\right)=(2 \mathrm{hr}, 401 \mathrm{~Hz})$ and IGR J00291 + 5934 at $(2.5 \mathrm{hr}, 599 \mathrm{~Hz})$, which show short-term spin-up and long-term spin-down, respectively (see the text).

(A color version of this figure is available in the online journal.)

\section{DISCUSSION}

We can now understand the observed population of AMSPs (especially the absence of sources at low spin and long orbital period) as a result of the evolution of $v_{\mathrm{s}}$ and $P_{\mathrm{orb}}$, depending on the process (magnetic braking, mass accretion, and gravitational radiation) with the shortest timescale. AMSPs born at low $v_{\mathrm{s}}$ and long $P_{\text {orb }}$ very quickly spin up to high $v_{\mathrm{s}}$. Then on much longer timescales, these fast spinning sources slow down and their orbits decrease. Once mass accretion ceases, there is no longer a spin-up torque; the binary then contains a rotationpowered millisecond pulsar that can move into the (former) spin-up region in Figure 3 or 4 by spinning down (as a result of gravitational wave or electromagnetic radiation) or expanding its orbit (see Figure 1).

We utilize two very different prescriptions for angular momentum loss due to magnetic braking and find that we can vacate the low spin, long orbital period spin-up region for reasonable parameter choices in both cases. We note a slight preference for the results obtained using Equations (4) and (5), as compared to Equations (6) and (7). Future observations, for example, measurement of the mass accretion rate dependence on orbital period, could distinguish between the two models. There are presently six AMSPs with unknown $P_{\text {orb }}$, three of which have $v_{\mathrm{s}}<363 \mathrm{~Hz}$. Measurement of a long orbital period for these systems or discovery of new systems at the relatively low $v_{\mathrm{s}}$ $\left(100 \mathrm{~Hz} \lesssim v_{\mathrm{s}} \lesssim 400 \mathrm{~Hz}\right)$ and long $P_{\text {orb }}$ would challenge the scenario proposed here. Clearly, more detailed studies of short orbital period systems are required to address the trend seen, as well as to account for spin evolution in binary evolution models (see, e.g., Podsiadlowski et al. 2002; Lamb \& Yu 2005; Ferrario \& Wickramasinghe 2007).

The most rapidly rotating millisecond radio pulsar (Hessels et al. 2006) and AMSP have $v_{\mathrm{s}}=716 \mathrm{~Hz}$ and $620 \mathrm{~Hz}$, respectively. These frequencies are far below the theoretical maximum (at $>1 \mathrm{kHz}$ ), above which the centrifugal force causes mass-shedding (Cook et al. 1994; Haensel et al. 1999). It is thought that angular momentum loss from gravitational radiation could be responsible for a spin limit below the massshedding maximum (Wagoner 1984; Bildsten 1998; Andersson et al. 1999; Melatos \& Payne 2005). However, theoretical predictions for this limit are very uncertain, with only the current gravitational wave searches (Abbott et al. 2010) and X-ray observations (Chakrabarty et al. 2003) serving as constraints. In our scenario, the observed position of AMSPs in Figures 3 and 4 relative to the different evolution regions is suggestive that the amplitude of the mass quadrupole that produces gravitational radiation is $\varepsilon \gtrsim 10^{-9}$. At lower $\varepsilon$, spin-down by gravitational radiation becomes irrelevant (see Equations (12) and (14)), and all AMSPs would be in spin-up or orbit expansion or decay, contrary to what is seen (Hartman et al. 2009, 2011; Papitto et al. 2010; Patruno 2010); note that spin-down by electromagnetic radiation gives a similar region in Figures 3 and 4 only if $B \gtrsim 10^{9} \mathrm{G}$. Our estimated quadrupole ellipticity is far below the theoretical maximum (Horowitz \& Kadau 2009) of $4 \times 10^{-4}$ and below the current limit of $7 \times 10^{-8}$ set by gravitational wave detectors (Abbott et al. 2010). Though observationally challenging (see, e.g., Watts et al. 2008), future searches by Advanced LIGO or the proposed Einstein Telescope (Punturo et al. 2010; Andersson et al. 2011) could provide direct evidence for the evolutionary scenario outlined here.

The authors thank C. O. Heinke, D. I. Jones, and the anonymous referee for comments that contributed to improvements to the manuscript. W.C.G.H. appreciates the use of the computer facilities at the Kavli Institute for Particle Astrophysics and Cosmology. W.C.G.H. and N.A. acknowledge support from the Science and Technology Facilities Council (STFC) in the United Kingdom.

\section{REFERENCES}

Abbott, B. P., et al. 2010, ApJ, 713, 671

Alpar, M. A., Cheng, A. F., Ruderman, M. A., \& Shaham, J. 1982, Nature, 300, 728

Andersson, N., Ferrari, V., Jones, D. I., Kokkotas, K. D., Krishnan, B., Read,

J. S., Rezzolla, L., \& Zink, B. 2011, Gen. Rel. Grav., 43, 409

Andersson, N., Kokkotas, K. D., \& Stergioulas, N. 1999, ApJ, 516, 307

Andronov, N., Pinsonneault, M., \& Sills, A. 2003, ApJ, 582, 358

Archibald, A., et al. 2009, Science, 324, 1411

Barret, D., \& Olive, J.-F. 2002, ApJ, 576, 391

Bhattacharya, D. 1995, in X-ray Binaries, ed. W. H. G. Lewin, J. van Paradijs,

\& E. P. J. van den Heuvel (Cambridge: Cambridge Univ. Press), 233

Bildsten, L. 1998, ApJ, 501, L89

Chaboyer, B., Demarque, P., \& Pinsonneault, M. H. 1995, ApJ, 441, 865

Chakrabarty, D., \& Morgan, E. H. 1998, Nature, 394, 346

Chakrabarty, D., Morgan, E. H., Muno, M. P., Galloway, D. K., Wijnands, R., van der Klis, M., \& Markwardt, C. B. 2003, Nature, 424, 42

Cook, G. B., Shapiro, S. L., \& Teukolsky, S. A. 1994, ApJ, 423, L117

Cumming, A., Zweibel, E., \& Bildsten, L. 2001, ApJ, 557, 958

Davidson, K., \& Ostriker, J. P. 1973, ApJ, 179, 585

Di Salvo, T., Burderi, L., Riggio, A., Papitto, A., \& Menna, M. T. 2008, MNRAS, 389, 1851

Falanga, M., et al. 2005, A\&A, 444, 15

Ferrario, L., \& Wickramasinghe, D. 2007, MNRAS, 375, 1009

Galloway, D. K. 2008, in AIP Conf. Proc. 983, 40 Years of Pulsars, ed. C. G. Bassa et al. (Melville, NY: AIP), 510 
Galloway, D. K., Muno, M. P., Hartman, J. M., Psaltis, D., \& Chakrabarty, D. 2008, ApJS, 179, 360

Gladstone, J., Done, C., \& Gierliński, M. 2007, MNRAS, 378, 13

Haensel, P., Lasota, J. P., \& Zdunik, J. L. 1999, A\&A, 344, 151

Hartman, J. M., Galloway, D. K., \& Chakrabarty, D. 2011, ApJ, 726, 26

Hartman, J. M., Patruno, A., Chakrabarty, D., Markwardt, C., Morgan, E. H., van der Klis, M., \& Wijnands, R. 2009, ApJ, 702, 1673

Hartman, J. M., et al. 2008, ApJ, 675, 1468

Hessels, J. W., Ransom, S. M., Stairs, I. H., Freire, P. C. C., Kaspi, V. M., \& Camilo, F. 2006, Science, 311, 1901

Horowitz, C. J., \& Kadau, K. 2009, Phys. Rev. Lett., 102, 191102

Kaaret, P., Morgan, E. H., Vanderspek, R., \& Tomsick, J. A. 2006, ApJ, 638, 963

King, A. R., Kolb, U., \& Burderi, L. 1996, ApJ, 464, L127

Lamb, F. K., \& Yu, W. 2005, in ASP Conf. Ser. 328, Binary Radio Pulsars, ed. F. A. Rasio \& I. H. Stairs (San Francisco, CA: ASP), 299

Ma, B., \& Li, X.-D. 2009, ApJ, 691, 1611

Maccarone, T. J., \& Coppi, P. S. 2003, MNRAS, 338, 189

Manchester, R. N., Hobbs, G. B., Teoh, A., \& Hobbs, M. 2005, AJ, 129, 1993

Melatos, A., \& Payne, D. J. B. 2005, ApJ, 623, 1044

Paczyński, B. 1971, ARA\&A, 9, 183

Papitto, A., Riggio, A., Burderi, L., Di Salvo, T., D’Aí, A., \& Iaria, R. 2011, A\&A, 528, A55
Patruno, A. 2010, ApJ, 722, 909

Podsiadlowski, Ph., Rappaport, S., \& Pfahl, E. D. 2002, ApJ, 565, 1107

Punturo, M., et al. 2010, Class. Quantum Grav., 27, 084007

Radhakrishnan, V., \& Srinivasan, G. 1982, Curr. Sci., 51, 1096

Sills, A., Pinsonneault, M. H., \& Terndrup, D. M. 2000, ApJ, 534, 335

Smarr, L. L., \& Blandford, R. 1976, ApJ, 207, 574

Strohmayer, T., \& Bildsten, L. 2006, in Compact Stellar X-ray Sources, ed W. H. G. Lewin \& M. van der Klis (Cambridge: Cambridge Univ. Press), 113

Strohmayer, T. E., Zhang, W., Swank, J. H., Smale, A., Titarchuk, L, Day, C., \& Lee, U. 1996, ApJ, 469, L9

Verbunt, F., \& van den Heuvel, E. P. J. 1995, in X-ray Binaries, ed. W. H. G. Lewin, J. van Paradijs, \& E. P. J. van den Heuvel (Cambridge: Cambridge Univ. Press), 457

Verbunt, F., \& Zwaan, C. 1981, A\&A, 100, L7

Wagoner, R. V. 1984, ApJ, 278, 345

Watts, A. L., Krishnan, B., Bildsten, L., \& Schutz, B. F. 2008, MNRAS, 389, 839

Wijnands, R., \& van der Klis, M. 1998, Nature, 394, 344

Wijnands, R., van der Klis, M., Homan, J., Chakrabarty, D., Markwardt, C. B., \& Morgan, E. H. 2003, Nature, 424, 44

Yungelson, L. R., \& Lasota, J.-P. 2008, A\&A, 488, 257 